[9] -, Hardy's inequality with weights in two dimensions, Notices Amer. Math. Soc. 29 (6) (1982), 538.

[10] -, Weighted norm inequalities for the Fourier transform, Trans. Amer. Math. Soc. 276 (1983), 729-742.

[11] B. Muckenhoupt and R. L. Wheeden, Two weight function norm inequalities for the Hardy-Littlewood maximal function and the Hilbert transform, Studia Math. 55 (1976), 279-294.

[12] G. Okikiolu, Aspects of the Theory of Bounded Integral Operators in $L^{p}$ Spaces, Academic Press, New York, N. Y. 1971.

[13] F. Riesz, Sur une inégalité intégrale, J. London Math. Soc. 5 (1930), 162-168.

[14] E. Sawyer, A characterization of a two-weight norm inequality for maximal operators, Studia Math. 75 (1982), 1-11.

[15] -, Weighted Lebesgue and Lorentz norm inequalities for the Hardy operator, Trans. Amer. Math. Soc. 281 (1984), 329-337.

[16] E. M. Stein and G. Weiss, Introduction to Fourier Analysis on Euclidean Spaces, Princeton Univ. Press, Princeton, N. J. 1971.

[17] G. Talenti, Osservazioni sopra una classe di disuguaglianze, Rend. Sem. Mat. Fis. Milano 39 (1969), 171-185.

[18] G. Tomasselli, A class of inequalities, Boll. Un. Mat. Ital. 21 (1969), 622-631.

DEPARTMENT OF MATHEMATICAL SCIENCES

MCMASTER UNIVERSITY

Hamilton, Ontario, Canada

Received June 3, 1983

\section{Generalizations of Calderón-Zygmund operators}

by

KÓZÔ YABUTA (Mito)

Dedicated to Professor Sigeru Mizohata on the occasion of his sixtieth birthday

Abstract. Calderón-Zygmund operators are introduced by R. Coifman and Y. Meyer to treat systematically the classical Calderón-Zygmund singular integrals, commutators of Calderón, and some classes of pseudo-differential operators. In this note we generalize the notion of Calderón-Zygmund operators and apply it to the study of, for example, weighted norm inequalities for certain classes of pseudo-differential operators, treated by Coifman and Meyer, and recently G. Bourdaud. We also refer to a recent work of David and Journé on the $L^{2}$-boundedness criterion for operators of the Calderón-Zygmund type:

1. Introduction. Calderón-Zygmund operators are introduced by R. Coifman and Y. Meyer [5] to treat systematically the classical CalderónZygmund singular integrals, the commutators of Calderón and some classes of pseudo-differential operators, etc., and are further developed by Journé [10]. They are used in many places, [3], [6], [7], [16], [17], etc. In this note we introduce two classes of operators which are generalizations of CalderónZygmund operators, and apply them to some classes of pseudo-differential operators considered in [1], [5] and [13].

A Calderón-Zygmund operator defined by Coifman and Meyer, is as follows: Let $T$ be a bounded operator from the class $\mathscr{S}\left(\boldsymbol{R}^{n}\right)$ of Schwartz functions to its dual $\mathscr{S}^{\prime}\left(\boldsymbol{R}^{\prime \prime}\right)$, satisfying the following two conditions.

(A-1) There exists $C>0$ such that for any $f \in C_{0}^{\infty}\left(\boldsymbol{R}^{n}\right)$

$$
\|T f\|_{L^{2}\left(\boldsymbol{m}^{\prime \prime}\right)} \leqslant C\|f\|_{L^{2}\left(\boldsymbol{m}^{n}\right)}
$$

(A-2) There exist a continuous function $K(x, y)$ defined on $\Omega=\boldsymbol{R}^{n} \times$ $\times \boldsymbol{R}^{\top} \backslash\{(x, y) ; x=y\}$ and $C_{\boldsymbol{K}}>0$ such that

(1.1) for all $(x, y) \in \Omega$

$$
|K(x, y)| \leqslant C_{K}|x-y|^{-n}
$$

Partly supported by the Grant-in-Aid for Co-operative Research (A-57340004), the Ministry of Education, Science and Culture, Japan., 
(1.2) for all $x, x_{0}, y$ with $2\left|x_{0}-x\right|<|x-y|$,

$$
\left|K\left(x_{0}, y\right)-K(x, y)\right|+\left|K\left(y, x_{0}\right)-K(y, x)\right| \leqslant C_{K} \frac{\left|x_{0}-x\right|}{|x-y|^{n+1}} ;
$$

(1.3) for all $f \in C_{0}^{\infty}\left(\boldsymbol{R}^{n}\right)$,

$$
T f(x)=\int_{\boldsymbol{R}^{n}} K(x, y) f(y) d y \quad \text { a.e. on }[\operatorname{supp} f]^{\mathrm{c}} \text {. }
$$

Then $T$ is said to be a Calderón-Zygmund operator (we abbreviate it to $\mathrm{CZO}$ ). A function $K$ satisfying (1.1) and (1.2) is said to be a standard kernel.

We recall the typical and important properties of CZO. To do so, we recall some notations. For $f \in L_{\text {loc }}^{1}\left(\boldsymbol{R}^{n}\right)$, let

$$
m_{I} f=|I|^{-1} \int_{I} f(x) d x \quad \text { and } \quad\|f\|_{\mathrm{BMO}}=\sup |I|^{-1} \int_{I}\left|f(x)-m_{I} f\right| d x,
$$

where the supremum is taken over all cubes $I$ with sides.parallel to the coordinate axes, and $|I|$ denotes the Lebesgue measure of $I$. Then the space of functions of bounded mean oscillation is defined by $\operatorname{BMO}\left(\boldsymbol{R}^{n}\right)$ $=\left\{f \in L_{\text {loc }}^{1}\left(\boldsymbol{R}^{n}\right) ;\|f\|_{\text {Вмо }}<\infty\right\}$ with the seminorm $\|f\|_{\text {ВMO }}$. The real Hardy space $H^{1}\left(\boldsymbol{R}^{n}\right)$ is the space of all $L^{1}\left(\boldsymbol{R}^{n}\right)$-functions $f$ with $R_{j} f \in L^{1}\left(\boldsymbol{R}^{n}\right)(j$ $=1,2, \ldots n)$, endowed with the norm

$$
\|f\|_{H^{1}\left(\boldsymbol{R}^{n}\right)}=\|f\|_{L^{1}\left(\boldsymbol{R}^{\left.\boldsymbol{N}^{\prime}\right)}\right.}+\sum_{j}\left\|R_{j} f\right\|_{\mathcal{L}^{1}\left(\boldsymbol{R}^{\prime \prime}\right)}
$$

where $R_{j} f$ is the Riesz transform of $f$, i.e., its Fourier transform $\left(R_{j} f\right)(\xi)$ is given by $-i \xi_{j}|\xi|^{-1} \hat{f}(\xi)$. (See [8], [10] for the spaces BMO and $H^{1}$.) We recall also the Muckenhoupt $A_{p}$ weight classes. For $1<p<\infty$, a nonnegative function $w(x) \in L_{\text {loc }}^{1}\left(\boldsymbol{R}^{n}\right)$ is said to be in $A_{p}\left(\boldsymbol{R}^{n}\right)$ if

$$
|w|_{A_{p}}=\sup _{I}\left(|I|^{-1} \int_{I} w d x\right)\left(|I|^{-1} \int_{I} w^{-1 /(p-1)} d x\right)^{p-1}<\infty
$$

where the supremum is taken over all cubes $I$ in $\boldsymbol{R}^{n}$. Similarly a nonnegative function $w \in L_{\text {loc }}^{1}\left(\boldsymbol{R}^{n}\right)$ is said to be in $A_{1}\left(\boldsymbol{R}^{n}\right)$ if

$$
|w|_{A_{1}}=\sup _{I}\left(|I|^{-1} \int_{I} w d x\right)(\underset{x \in I}{\operatorname{essinf}} w(x))^{-1}<\infty .
$$

(See [4], [9], [10] for the basic properties of $A_{p}$ )

Now, the well-known important properties of $\mathrm{CZO}$ are the following:

(P-1) For any weight $w \in A_{p}\left(\boldsymbol{R}^{n}\right)(1<p<\infty)$,

$$
\|T f\|_{L^{p}(w d x)} \leqslant C(p, w)\|f\|_{L^{p}(w d x)} ;
$$

(P-2) For any weight $w \in A_{1}\left(\boldsymbol{R}^{n}\right)$,

$$
w\left(\left\{x \in \boldsymbol{R}^{n} ;|T f(x)|>\lambda\right\}\right) \leqslant \frac{C(w)}{\lambda}\|f\|_{L^{1}(w d x)} ;
$$

(P-3) $\|T f\|_{L^{1}\left(R^{n}\right)} \leqslant C_{1}\|f\|_{H^{1}\left(R^{n}\right)}$;

(P-4) $\|T f\|_{\mathrm{BMO}\left(\boldsymbol{R}^{n}\right)} \leqslant C_{\infty}\|f\|_{L^{\infty}\left(\boldsymbol{R}^{n}\right)}$; where

(P-5) (P-1) and (P-2) hold for the truncated maximal operator $T_{*}$ of $T$,

$$
T_{*} f(x)=\sup _{\varepsilon>0}\left|\int_{|x-y|>\varepsilon} K(x, y) f(y) d y\right| .
$$

In the next section we generalize the notion of $\mathrm{CZO}$ so that it has the above properties (P-1) through (P-5). In Section 3 we apply the results in Section 2 to certain classes of pseudo-differential operators, considered in [1], [5], [13] and [14]. Our Theorems 3.1 and 3.2 comprise a recent result of Nishigaki [15]. In the last section we generalize the notion of standard kernel, so that the recent result of David and Journé holds true, i.e., their criterion for $L^{2}$-boundedness works.

In this paper, $C_{0}^{\infty}\left(\boldsymbol{R}^{n}\right)$ is the set of all infinitely differentiable functions with compact support in $\boldsymbol{R}^{n}$ and $L^{p}\left(\boldsymbol{R}^{n}\right)$ is the usual $L^{p}$-space with respect to the Lebesgue measure on $\boldsymbol{R}^{n}$, endowed with the norm $\|f\|_{L^{p}\left(\boldsymbol{R}^{n}\right)}$ $=\left(\int|f(x)|^{p} d x\right)^{1 / p}$. For a weight function $w(x)$ we similarly define $L^{p}(w d x)$, and ${ }^{\pi^{n}} w(E)$ denotes the measure of the set $E$ with respect to $w(x) d x$.

Finally we note that the letter $C$ will denote a constant which may vary from line to line.

2. Calderón-Zygmund operators of type $\omega(t)$ and of type $(\log , \omega(t))$. Following Coiîman and Meyer we introduce two notions of "standard kernel".

DefinITION 2.1. Let $\omega(t)$ be a nonnegative nondecreasing function on $\boldsymbol{R}_{+}=(0, \infty)$ with $\int_{0}^{1} \omega(t) t^{-1} d t<\infty$. A continuous function $K(x, y)$ on $\Omega$ $=\boldsymbol{R}^{n} \times \boldsymbol{R}^{\eta} \backslash\left\{(x, x) ; x \in \boldsymbol{R}^{n}\right\}$ is said to be a standard kernel of type $\omega(t)$ if the following are satisfied:

$$
|K(x, y)| \leqslant C|x-y|^{-n}
$$

(2.2) $\left|K(x, y)-K\left(x_{0}, y\right)\right|+\left|K(y, x)-K\left(y, x_{0}\right)\right| \leqslant C\left|x_{0}-y\right|^{-n} \omega\left(\frac{\left|x_{0}-x\right|}{\left|y-x_{0}\right|}\right)$,

for all $x, x_{0}, y$ with $2\left|x-x_{0}\right|<\left|y-x_{0}\right|$.

Definition 2.2. Let $\omega(t)$ be the same as above. A continuous function $K(x, y)$ on $\Omega$ is said to be a standard kernel of type $(\log , \omega(t))$ if it satisfies (2.1) and

$\left(2.2^{\prime}\right)$. For all $x, x_{0}, y$ with $2\left|x-x_{0}\right|<\left|y-x_{0}\right|$, the left-hand side of (2.2) is 
bounded by

$$
C\left[\left|x_{0}-y\right|^{-n} \omega\left(\frac{\left|x_{0}-x\right|}{\left|y-x_{0}\right|}\right)+G\left(x, x_{0}, y\right)\right]
$$

where $G\left(x, x_{0}, y\right)$ is a function satisfying

$$
\begin{aligned}
& G\left(x, x_{0}, y\right) \\
& \leqslant \begin{cases}\left|x_{0}-y\right|^{-n}\left(\log \frac{2}{\left|x-x_{0}\right|}\right)^{-1} & \text { if }\left|x-x_{0}\right| \leqslant 1 \text { and }\left|x_{0}-y\right| \leqslant 2 \sqrt{n} \\
\left|x_{0}-y\right|^{-n} \omega\left(\frac{\max \left(\left|x-x_{0}\right|, 1\right)}{\left|x_{0}-y\right|}\right) & \text { if }\left|y-x_{0}\right| \geqslant 1\end{cases}
\end{aligned}
$$

For a standard kernel $K,|K|_{\mathrm{CZ}}$ denotes the infimum of $C$ in (2.1) and (2.2) (or $\left(2.2^{\prime}\right)$ ).

Definition 2.3. Let $\omega(t)$ be the same as in Definition 2.1. A linear operator $T$ from $\mathscr{S}\left(\boldsymbol{R}^{n}\right)$ to $\mathscr{S}^{\prime}\left(\boldsymbol{R}^{n}\right)$ is said to be a Calderón-Zygmund operator of type $\omega(t)$ (respectively, of type $(\log , \omega(t)))$ if the following conditions are satisfied:

$$
\text { (A-1) }\|T f\|_{\mathbf{L}^{2}\left(\boldsymbol{R}^{n}\right)} \leqslant \dot{C}\|f\|_{L^{2}\left(\boldsymbol{R}^{n}\right)}, \quad f \in C_{0}^{\infty}\left(\boldsymbol{R}^{n}\right)
$$

(A-2') There exists a standard kernel $K(x, y)$ of type $\omega(t)$ (respectively, of type $(\log , \omega(t)))$ such that for all $f \in C_{0}^{\infty}\left(\boldsymbol{R}^{n}\right)$

$$
T f(x)=\int K(x, y) f(y) d y \quad \text { a.e. on }[\operatorname{supp} f]^{c} .
$$

We abbreviate Calderón-Zygmund operators of type $\omega(t)$ (or of type $(\log , \omega(t)))$ to $\mathrm{CZO}$ of type $\omega(t)(\operatorname{or}(\log , \omega(t)))$, or simply CZO. For a $T \in \mathrm{CZO},|T|_{\mathrm{CZ}}$ will denote the infimum of $C$ in (A-1) plus $|K|_{\mathrm{CZ}}$.

Now our main result is:

THEOREM 2.4. Let $\omega(t)$ be a nonnegative nondecreasing function on $(0, \infty)$ with $\int_{0}^{1} \omega(t) t^{-1} d t<\infty$. Let $T$ be a Calderón-Zygmund operator of type $\omega(t)$ (or of type $(\log , \omega(t)))$. Then $T$ has the continuity properties (P-1) through (P-5).

Let $I(a, r)$ denote the cube with sides parallel to the coordinate axes and with side length $r$ and center $a$. For any cube $Q=I(a, r), \bar{Q}$ denotes the cube $I(a,(4 \sqrt{n}+1) r)$. Then, by the arguments in Journe [10] and CoifmanMeyer [5], the conclusions in the theorem follow from the following three estimates:
(2.3) For all $Q=I(x, r)$ and all $f \in L_{\mathrm{loc}}^{1}\left(\boldsymbol{R}^{n}\right)$,

$$
\int_{Q \backslash Q}|K(x, y) f(y)| d y \leqslant C M(f)(x),
$$

where $M(f)(x)=\sup _{x \in I}|I|^{-1} \int_{I}|f(y)| d y \quad$ (the Hardy-Littlewood maximal function);

(2.4) For all cubes $Q$, all $x, x_{0} \in Q$ and all $f \in L_{\mathrm{loc}}^{1}\left(\boldsymbol{R}^{n}\right)$,

$$
\int_{Q^{c}}\left|K(x, y)-K\left(x_{0}, y\right)\right||f(y)| d y \leqslant C M(f)\left(x_{0}\right) ;
$$

(2.5) For all cubes $Q$, all $y_{0} \in Q$, all $f \in L_{\mathrm{loc}}^{1}\left(\boldsymbol{R}^{n}\right)$, and all $w \in A_{1}\left(\boldsymbol{R}^{n}\right)$,

$$
\int_{Q^{c}} \int_{Q}\left|K(x, y)-K\left(x, y_{0}\right)\right||f(y)| w(x) d y d x \leqslant C(n, w) w(Q)|Q|^{-1} \int_{Q}|f(y)| d y .
$$

Now (2.3) follows immediately from (2.1). Instead of (2.4) and (2.5) we shall show a stronger inequality: for all cubes $Q$ and all $x, x_{0} \in Q$,

$$
\int_{\mathscr{Q}^{c}}\left|K(x, y)-K\left(x_{0}, y\right)\right||f(y)| d y \leqslant C \sup _{Q \subset I}|I|^{-1} \int_{I}|f(y)| d y .
$$

Once this is established, (2.4) is trivial, and taking $f=w$ in (2.6) and noting that

$$
|I|^{-1} \int_{I} w(y) d y \leqslant|w|_{A_{1}} \underset{x \in I}{\operatorname{essinf}} w(x) \leqslant|w|_{A_{1}}|Q|^{-1} \int_{Q} w(y) d y
$$

(by the definition of $A_{1}$ and $Q \subset I$ ), we get

$$
\int_{\mathscr{Q}^{\mathrm{C}}}\left|K(x, y)-K\left(x_{0}, y\right)\right| w(y) d y \leqslant C|w|_{\boldsymbol{A}_{1}}|Q|^{-1} w(Q) .
$$

Hence, from the form of $K(x, y)$, interchanging the roles of $x$ and $y$, we obtain (2.5). So we have only to show (2.6).

Proof of (2.6). We shall prove this for the case where $\mid K(x, y)$ $-K\left(x_{0}, y\right) \mid \leqslant G\left(x, x_{0}, y\right)$. In the same way as in the following Case 1 one can handle the case of Definition 2.1. One can complete the case of Definition 2.2 by combining the two cases. Now let $Q=I(a, r)$ be an arbitrary cube.

Case 1: $\sqrt{n} r \geqslant 1$. If $\dot{y} \in \bar{Q}^{\mathrm{c}}$, then $\left|x_{0}-y\right| \geqslant 2 \sqrt{n} r \geqslant 2$ and $2\left|x_{0}-x\right|$ $<\left|x_{0}-y\right|$. Hence by $\left(2.2^{\prime}\right)$

$$
\left|K(x, y)-K\left(x_{0}, y\right)\right| \leqslant C\left|x_{0}-y\right|^{-n} \omega\left(\sqrt{n} r /\left|x_{0}-y\right|\right) .
$$


So

$$
\begin{aligned}
& \int_{\bar{Q}^{\mathrm{c}}}\left|K(x, y)-K\left(x_{0}, y\right)\right||f(y)| d y \\
& \quad \leqslant \int_{I\left(x_{0}, 4 \sqrt{n} r\right)^{\mathrm{c}}} \ldots d y \\
& \leqslant \sum_{j=0}^{\infty} \int_{I\left(x_{0}, 2^{j+3} \sqrt{n} r\right) \backslash I\left(x_{0}, 2^{j+2} \sqrt{n} r\right)} \ldots d y \\
& \leqslant C \sum_{j=0}^{\infty} \frac{\omega\left(\sqrt{n} r /\left(2^{j+1} \sqrt{n} r\right)\right)}{\left(2^{j+1} \sqrt{n} r\right)^{n}} \int_{I\left(x_{0}, 2^{j+3}, \bar{n} r\right)}|f(y)| d y \\
& \leqslant 4^{n} C\left(\sum_{j=0}^{\infty} \omega\left(2^{-j-1}\right)\right) \sup _{Q \subset I}|I|^{-1} \int_{I}|f(y)| d y .
\end{aligned}
$$

The series in parenthesis above is equivalent to $\int_{0}^{1} \omega(t) t^{-1} d t$.

Case $2: \sqrt{n} r \leqslant 1$. In this case $\left|x-x_{0}\right|<\sqrt{n} r<1$ and $2\left|x_{0}-x\right|$
$<\left|y-x_{0}\right|$. Hence

$$
\begin{gathered}
\int_{\Phi^{\mathrm{c}}}\left|K(x, y)-K\left(x_{0}, y\right)\right||f(y)| d y \\
\leqslant \sum_{0 \leqslant j \leqslant-\log _{2}(\sqrt{n} r)} \int^{+\sum_{j=0}^{\infty} \int_{Y\left(x_{0}, 2^{j+2}\right) \backslash I\left(x_{0}, 2^{j+1}\right)} \ldots d y} \ldots d y \\
=I_{1}+I_{2} .
\end{gathered}
$$

Then by the first inequality for $G\left(x, x_{0}, y\right)$ in $\left(2.2^{\prime}\right)$ we get

$$
\begin{aligned}
I_{1} & \leqslant \frac{C}{\log \left(2 /\left|x-x_{0}\right|\right)} \sum\left(2^{j+1} \sqrt{n} r\right)^{-n}: \int_{I\left(x_{0}, 2^{j+3} \sqrt{n} r\right)}|f(y)| d y \\
& \left.\leqslant C \sup _{Q \subset I}|I|^{-1} \int_{I}|f(y)| d y \quad \text { (by }\left|x-x_{0}\right|<\sqrt{n} r\right) .
\end{aligned}
$$

By the second inequality for $G$ in $\left(2.2^{\prime}\right)$ we get

$$
\begin{aligned}
I_{2} & \leqslant C \sum_{j=0}^{\infty} \frac{\omega\left(2^{-j}\right)}{2^{j n}} \int_{I\left(x_{0}, 2^{j+2},\right.}|f(y)| d y \\
& \leqslant C\left(\sum_{j=0}^{\infty} \omega\left(2^{-j}\right)\right) \sup _{Q \subset I}|I|^{-1} \int_{I}|f(y)| d y .
\end{aligned}
$$

This completes the proof of (2.6) and hence of Theorem 2.4.

Remark 2.5. As shown by Proposition 3.5 in the next section, Theorem 2.4 is sharp in the sense that the properties (P-1) through (P-5) hold.

Finally, in this section we note the following two properties of CZO's.

Proposition 2.6. Let $\omega(t)$ be the same as in Theorem 2.4. Suppose that $T$ is a bounded linear operator on $L^{2}\left(\boldsymbol{R}^{n}\right)$ and that there exists a sequence $\left(T_{j}\right)_{j \in N}$ of CZO's of type $\omega(t)$ (respectively, $(\log , \omega(t))$ ) such that
(i) $\left|T_{j}\right|_{(\%}$ is uniformly bounded in $j$;
(ii) $\lim \left\|T_{j} f-T f\right\|_{L^{2}\left(\boldsymbol{R}^{n}\right)}=0$ for all $f \in C_{0}^{\infty}\left(\boldsymbol{R}^{n}\right)$.

Then $T$ is a CZO of type $\omega(t)$ (respectively, $(\log , \omega(t))$ ).

Proof. Using the equicontinuity and uniform boundedness of $K_{j}$ on every compact set in $\Omega$, one can prove the above quite similarly to $[5, \mathrm{pp}$. 83-84].

Proposition 2.7. Let $T$ he a CZO and $b \in \mathrm{BMO}\left(\boldsymbol{R}^{n}\right)$. Then the commutator of $T$ and the operator of multiplication by $b,[b, T]$, is bounded on all $L^{p}(w d x)$, for $1<p<\infty$ and $w \in A_{p}$.

The proof in $[10$, p. 60$]$ works without change.

3. Pseudo-differential operators. In this section we assume that $\omega(t)$ is a nonnegative, nondecreasing and concave function on $(0, \infty)$ and $\Omega(t)$ is a positive function on $(0, \infty)$ satisfying the following property: for every $c>1$ there exists $A(c)>0$ such that $t / c \leqslant s \leqslant c t$ implies $\Omega(s) \leqslant A(c) \Omega(t)$.

Let $\sigma(x, \xi)$ be a sufficiently smooth function defined on $\boldsymbol{R}^{n} \times \boldsymbol{R}^{n}$. The pseudo-differential operator $(\psi$. d. o.) with symbol $\sigma$ is defined on the Schwartz class by the formula

$$
\sigma(x, D) f(x)=\operatorname{op}(\sigma) f(x)=(2 \pi)^{-n} \int_{\boldsymbol{R}^{n}} e^{i x \cdot \xi} \sigma(x, \xi) \hat{f}(\xi) d \xi,
$$

(where $\hat{f}(\xi)=\int e^{-i x \cdot \xi} f(x) d x$ is the Fourier transform of $f$ ).

Coifman and Meyer [5] showed that if $\sigma \in S_{1,0}^{0}$ (Hörmander's class) then $\sigma(x, D)$ is a Calderón-Zygmund operator. They also showed the $L^{p}$ boundedness of $\sigma(x, D)$ for certain classes of $\psi$. d. o. with less regular symbol. Bourdaud [1] has developed the subject further. We shall show in 
this section that for a subclass of $\psi$. d. o., $\sigma(x, D)$ is a CZO in the sense of Section 2 , so that, for example, weighted $L^{p}$-norm inequalities hold in this case as in the $S_{1,0}^{0}$ case in Miller [12] and as in Nishigaki's case [15].

Our results are the following two theorems. Though the first one is contained in the second in the sense that properties (P-1) through (P-5) hold, we hope that it would have its own sense; at least a CZO of type $\omega(t)$ is simpler than a CZO of type $(\log , \omega(t))$.

TheOREM 3.1. Let $0<a \leqslant 1, \Omega(t)$ be nondecreasing, $\omega(1)>0$, $\int_{0}^{1} \omega^{a}(t) t^{-1} d t<\infty$, and $\omega^{1-a}(t) \Omega\left(t^{-1}\right)$ be bounded on $(0,1]$. Suppose that $\sigma(x, \xi) \in C\left(\boldsymbol{R}^{n} \times \boldsymbol{R}^{n}\right)$ satisfies

$$
\left|\partial_{\xi}^{\alpha} \sigma(x, \xi)\right| \leqslant C_{\alpha}(1+|\xi|)^{-|\alpha|}, \quad|\alpha| \leqslant n+2 ;
$$

(3.2) $\left|\partial_{\xi}^{\alpha} \sigma(x+h, \xi)-\partial_{\xi}^{\alpha} \sigma(x, \xi)\right| \leqslant C_{\alpha} \omega(|h|) \Omega(|\xi|)(1+|\xi|)^{-|\alpha|}, \quad|\alpha| \leqslant n+1$,

where $\alpha$ is a multiindex and $|\alpha|=\alpha_{1}+\ldots+\alpha_{n}$. Then $\sigma(x, D)$ is a CalderónZygmund operator of type $\omega^{a}(t)$.

THEOREM 3.2. Let $0<a \leqslant 1, \Omega(t)$ be nondecreasing, $\omega^{a}(t)=O\left(\left(\log \frac{1}{t}\right)^{-1}\right)$ as $t \rightarrow 0$, and $\omega^{1-a}(t) \Omega\left(t^{-1}\right)$ be bounded on $(0,1]$. Suppose $\sigma(x, \xi)$ satisfies (3:1) and (3.2). Then $\sigma(x, D)$ is a Calderón-Zygmund operator of type $(\log , t)$.

Proof of Theorem 3.1. It is known that $\sigma(x, D)$ is bounded on $L^{2}\left(\boldsymbol{R}^{n}\right)$ provided $\sum_{j=0}^{\infty} \omega^{2}\left(2^{-j}\right) \Omega^{2}\left(2^{j}\right)<\infty$. (For $L^{2}\left(\boldsymbol{R}^{n}\right)$-boundedness one needs (3.1) and (3.2) only for $|\alpha| \leqslant n+1$, see [1] and [5].) This condition is satisfied in our case, because $\omega\left(2^{-j}\right) \Omega\left(2^{j}\right) \leqslant C \omega^{a}\left(2^{-j}\right)$ and $\int_{0}^{1} \omega^{a}(t) t^{-1} d t<\infty$. Using Proposition 2.6 (if necessary) and a standard argument, we may assume that the support of $\sigma(x, \xi)$ is contained in a set compact with respect to $\xi$. Therefore for $f \in C_{0}^{\infty}\left(\boldsymbol{R}^{n}\right)$

$$
\sigma(x, D) f(x)=\int K(x, y) f(y) d y,
$$

where

$$
K(x, y)=(2 \pi)^{-n} \hat{\sigma}(x, y-x)=(2 \pi)^{-n} \int_{\boldsymbol{R}^{n}} e^{-i(y-x) \cdot \xi} \sigma(x, \xi) d \xi .
$$

Thus we have only to check that this kernel $K(x, y)$ satisfies conditions (2.1) and (2.2) for $\omega$ replaced by $\omega^{a}(t)$. (2.1) and the estimate

$$
\left|K(y, x)-K\left(y, x^{\prime}\right)\right| \leqslant C \frac{\left|x-x^{\prime}\right|}{|x-y|^{n+1}} \quad\left(\left|x-x^{\prime}\right|<|x-y| / 2\right)
$$

are already known in Journé [10, pp. 65-66], under our assumption (3.1).
Since $\omega(1)>0$ and $\omega(t)$ is concave, $\omega^{a}(t)$ is also concave and $t<C \omega^{a}(t)(0$ $<t \leqslant 1$ ). So, it suffices to prove that for $|h|<|y| / 2$

$$
|\hat{\sigma}(x+h, y)-\hat{\sigma}(x, y)| \leqslant C|y|^{-n} \omega^{a}(|h| /|y|) .
$$

Let now $\psi(\xi) \in C_{0}^{\infty}\left(\boldsymbol{R}^{n}\right)$ be a radial function with support in $\{1 / 2<|\xi|$ $<2$ \} satisfying $\psi(\xi)=1$ on $3 / 4<|\xi|<1$ and $\Psi(\xi)=\sum_{j=1}^{\infty} \psi\left(2^{-j} \xi\right)=1$ on $|\xi|$ $\geqslant 2$. Let $\varphi(\xi)=1-\Psi(\xi)$. Put $\sigma_{0}(x, \xi)=\varphi(\xi) \sigma(x, \xi) \quad$ and $\quad \sigma_{j}(x, \xi)$ $=\psi_{j}(\xi) \sigma(x, \xi)(j=1,2, \ldots)$. Then $\sigma_{j}(x, \xi)$ satisfies (3.1) and (3.2) uniformly in $j$. Put

$$
L(x, h, y)=\hat{\sigma}(x+h, y)-\hat{\sigma}(x, y),
$$

and similarly define $L_{j}(x, h, y)$ in terms of $\sigma_{j}$. Then

$$
L_{j}=\int\left[\sigma_{j}(x+h, \xi)-\sigma_{j}(x, \xi)\right] e^{-i y \cdot \xi} d \xi .
$$

Hence, integration by parts and (3.2) yield for $j \geqslant 1$

$$
\begin{aligned}
|y|^{n+1}\left|L_{j}(x, h, y)\right| & \leqslant C \int_{2^{j-1}<|\xi|<2^{j+1}} \omega(|h|) \Omega(|\xi|)|\xi|^{-n-1} d \xi \\
& \leqslant C \omega(|h|) \Omega\left(2^{j}\right) 2^{-j} .
\end{aligned}
$$

The same inequality holds for $j=0$.

Case $1: 1 \leqslant|y|$. By the concavity of $\omega, \omega(t) / t$ is nonincreasing. Hence, one can easily show, by combining this with the nondecreasingness of $\omega$, that for $j \in N$

$$
\omega(|h|) \leqslant|y|^{a} 2^{j(1-a)} \omega^{a}(|h| /|y|) \omega^{1-a}\left(2^{-j}\right) \quad(|h| \leqslant 1)
$$

and

$$
\omega(|h|) \leqslant|y|^{a} 2^{j(1-a)}|h|^{1-a} \omega^{a}(|h| /|y|) \omega^{1-a}\left(2^{-j}\right) \quad(1 \leqslant|h|) .
$$

Therefore, for $|h|<|y| / 2$, we get

$$
\frac{\omega(|h|) \Omega\left(2^{j}\right)}{2^{j}|y|} \leqslant \omega^{a}(|h| /|y|) \omega^{1-a}\left(2^{-j}\right) \Omega\left(2^{j}\right) 2^{-a j}
$$

from which we get

$$
|L| \leqslant C \omega^{a}(|h| /|y|)|y|^{-n} .
$$

Case 2: $|y|<1$. Since $|h|<|y| / 2$, we have $\omega(|h|) \leqslant \omega^{a}(|h| /|y|) \omega^{1-a}(|y|)$. Notice that $\omega(t) / t$ is nonincreasing. Hence, if $1 \leqslant|y| 2^{j}$, we get $\omega(|y|) /|y|$ $\leqslant \omega\left(2^{-j}\right) 2^{j}$. Thus by (3.5) and the boundedness of $\omega^{1-a}(t) \Omega\left(t^{-1}\right)$ on $(0,1]$ we have

$$
\sum_{1 \leqslant 2^{j}|y|}\left|L_{j}\right| \leqslant C|y|^{-n} \omega^{a}(|h| /|y|) \sum_{1 \leqslant 2^{j}|y|}\left(2^{j}|y|\right)^{-a} \leqslant C \omega^{a}(|h| /|y|)|y|^{-n} .
$$


Now letting $j_{0}$ be the greatest $j$ satisfying $2^{j}|y| \leqslant 1$, we get

$=-1(\xi<-2)$. Let

$$
a(x)=\min \left(\left(\log \frac{1}{|x|}\right)^{-1}, 1 / \log 2\right) .
$$

For $0<\delta$, set

$$
\sigma_{\delta}(x, \xi)=(\operatorname{sgn} x) a^{\delta}(x) \sigma(\xi)
$$

Then

(i) if $1<\delta, \sigma_{\delta}(x, D)$ is a CZO of type $a^{\delta}(t)$;

(ii) if $\delta=1, \sigma_{\delta}(x, D)$ is a CZO of type $(\log , t)$;

(iii) if $0<\delta<1, \sigma_{\delta}(x, D)$ is not a CZO. There exists an $f \in L^{\infty}(R)$ with compact support such that $\sigma_{\delta}(x, D) f \notin \mathrm{BMO}(\boldsymbol{R})$.

Proof. One can easily show that $|a(x+h)-a(x)| \leqslant a(|h|)$. Hence, (i) is a consequence of Theorem 3.1. (ii) follows from Theorem 3.2 (with $\Omega(t)=1$ and $\left.\omega(t)=a^{\delta}(t)\right)$. We shall prove (iii). $\sigma(D)$ has the following form:

$$
\sigma(D)=c(H-\varphi * H), \quad \text { where } \hat{\varphi}(\xi)=1-\sigma(\xi) \operatorname{sgn} \xi \in C_{0}^{\infty}(R),
$$

and $H$ is the Hilbert transform. Since $H \chi_{(0,1)}=\pi \log |x /(x-1)|$, we have, putting $b_{\delta}(x)=(\operatorname{sgn} x) a^{\delta}(x)$,

$$
\begin{aligned}
\sigma_{\delta}(x, D) \chi_{(0,1)}(x)= & c b_{\delta}(x) \log |x|-c b_{\delta}(x) \log |x-1| \\
& -c(\varphi * \log |x /(x-1)|)(x) b_{\delta}(x) .
\end{aligned}
$$

The last two terms belong to BMO. However, if $0<\delta<1$, then, as is easily shown, $b_{\delta}(x) \log |x| \notin \mathrm{BMO}(\boldsymbol{R})$. This shows that $\sigma_{\delta}(x, D)$ is not a CZO. This completes the proof.

Remark 3.6. Let $K_{\delta}(x, y)=(2 \pi)^{-1} b_{\delta}(x) \hat{\sigma}(y-x)$ be the standard kernel associated with $\sigma_{\delta}(x, \xi)$. Then we can easily get $\left|K_{\delta}(x, y)\right| \leqslant C|x-y|^{-1}$, and for $\left|x-x_{0}\right| \leqslant\left|x_{0}-y\right| / 2$

$$
\begin{aligned}
& \left|K_{\delta}(y, x)-K_{\delta}\left(y, x_{0}\right)\right| \leqslant C\left|x-x_{0}\right| /\left|x_{0}-y\right|^{2}, \\
& \left|K_{\delta}(x, y)-K_{\delta}\left(x_{0}, y\right)\right| \leqslant C a^{\delta}\left(\left|x-x_{0}\right| /\left|x_{0}-y\right|\right)\left|x_{0}-y\right|^{-1} .
\end{aligned}
$$

The last inequality can be derived from the concavity and nondecreasingness of $a^{\delta}(t)(t>0)$ as in the proof of Theorem 3.1.

4. An $L^{2}$-boundedness criterion for operators with standard kernels. An operator $T$ from $\mathscr{S}\left(\boldsymbol{R}^{n}\right)$ to $\mathscr{S}^{\prime}\left(\boldsymbol{R}^{n}\right)$ has the weak cancellation property if for any bounded subset $E$ of $C_{0}^{\infty}\left(R^{n}\right)$ there exists a constant $C$ such'that for all $\varphi_{1}$ and $\varphi_{2} \in E$, all $x \in \boldsymbol{R}^{n}$ and $A>0$,

$$
\left|\left\langle T \varphi_{1}^{x, A}, \varphi_{2}^{x, A}\right\rangle\right| \leqslant C A^{n},
$$

Proposition 3.5. Let $\sigma(\xi) \in C^{\infty}(\boldsymbol{R})$ and $\sigma(\xi)=0(|\xi|<1),=1(2<\xi)$, and
a sense sharp. 
where $\varphi_{j}^{x, A}$ is defined by $\varphi_{j}^{x, A}(y)=\varphi_{j}((y-x) / A)$ for $j=1,2$ and $\langle$,$\rangle is the$ dual form for $\mathscr{S}$ and $\mathscr{S}^{\prime}$. David and Journé have recently proved the following in [7] and [7'].

TheOREM A. Let $T$ be a continuous operator from $\mathscr{S}\left(\boldsymbol{R}^{n}\right)$ to $\mathscr{S}^{\prime}\left(\boldsymbol{R}^{n}\right)$. Suppose there exists a standard kernel $K(x, y)$ of type $t^{\delta}$ (for some $\delta: 0$ $<\delta \leqslant 1)$ such that for all $f$ and $g \in C_{0}^{\infty}\left(\boldsymbol{R}^{n}\right)$ with disjoint supports,

$$
\langle T f, g\rangle=\iint K(x, y) f(y) g(x) d y d x .
$$

Then $T$ can be extended to a bounded operator from $L^{2}\left(\boldsymbol{R}^{n}\right)$ to itself if and only if the following three conditions are satisfied:
(i) $T 1 \in \mathrm{BMO}$,
(ii) $T^{*} 1 \in \mathrm{BMO}$,
(iii) $T$ has the weak cancellation property.

Here $T^{*}$ is the adjoint operator of $T$. For the details see [7]. Our remark on the above theorem is the following.

THEOREM 4.1. Let $\omega(t)$ be a nonnegative, nondecreasing and concave function on $(0, \infty)$ satisfying

$$
\int_{0}^{1} \omega^{1 / 3}(t) t^{-1} d t<\infty .
$$

Let $T$ be the same as in Theorem A, where $K(x, y)$ is a standard kernel of type $\omega(t)$. Then the conclusion in Theorem A holds true.

Proof. The "only if" part follows from Theorem 2.4. In order to prove the "if" part, one has only to follow the proof of David and Journe [7] word for word, replacing $t^{\delta}$ by $\omega(t)$. We only point out that fragment of our proof which differs most from their proof, where the condition $\int_{0}^{1} \omega^{1 / 3}(t) t^{-1} d t<\infty$ appears. It is Lemma 4.2 below. q.e.d.

Let $p(x)=\omega(1 /(1+|x|))(1+|x|)^{-n}$ and $p_{j}(x)=2^{-n j} p\left(2^{-j} x\right)$. Observe that

$$
\int_{\mathbb{R}^{n}} p_{j}(x) d x \leqslant C \int_{0}^{1} \omega(t) t^{-1} d t \leqslant C^{\prime} \int_{0}^{1} \omega^{1 / 3}(t) t^{-1} d t<\infty .
$$

For each $j \in N$, let $T_{j}$ be an integral operator which is defined by a kernel $K_{j}(x, y)$ such that

$$
\int K_{j}(x, y) d y=0 \text { for all } x \text {; }
$$$$
\int K_{j}(x, y) d x=0 \quad \text { for all } y \text {. }
$$

Then we have the following lemma, by which we can apply the CotlarKnapp-Stein lemma to the operators $\left\{T_{j}\right\}$.

LeMMA 4.2. Let $T_{j}$ be as above. Then there exists a constant $C>0$ such that for $(j, k) \in \boldsymbol{Z}^{2}$,

$$
\left\|T_{j} T_{k}^{*}\right\|_{L^{2}, L^{2}}+\left\|T_{j}^{*} T_{k}\right\|_{L^{2}, L^{2}} \leqslant C \omega^{2 / 3}\left(2^{-|j-k|}\right)
$$

where $\|\cdot\|_{L^{2}, L^{2}}$ denotes the operator norm from $L^{2}\left(\boldsymbol{R}^{n}\right)$ to $L^{2}\left(\boldsymbol{R}^{n}\right)$.

Proof. Let $K_{j, k}$ be the kernel of $T_{j}^{*} T_{k}$. Then the above results from the following estimates, by using the interpolation theorem:

$$
\int\left|K_{j, k}(x, y)\right| d y \leqslant C \omega^{2 / 3}\left(2^{-|j-k|}\right) \quad \text { for all } x,
$$

$$
\int\left|K_{j, k}(x, y)\right| d x \leqslant C \omega^{2 / 3}\left(2^{-|j-k|}\right) \text { for all } y,
$$

and similar estimates for the kernel of $T_{j} T_{k}^{*}$.

It suffices to prove the above estimates only when $j \leqslant k$, by virtue of (4.1) through (4.4). We only check (4.5), since the other is treated similarly. Using (4.1), (4.2) and (4.4), we get

$$
\left|K_{j, k}(x, y)\right| \leqslant \int p_{j}(z-x) \min \left(1,|z-x| / 2^{k}\right)\left[p_{k}(z-y)+p_{k}(x-y)\right] d z .
$$

Since $\int p_{k}(z-y) d y=C$, it suffices to show

$$
\int p_{j}(z) \min \left(1,|z| / 2^{k}\right) d z \leqslant C \omega^{2 / 3}\left(2^{-|j-k|}\right) .
$$

This can be shown as follows:

$$
\begin{aligned}
\int_{|z| \leqslant 2^{k}} p_{j}(z)|z| 2^{-k} d z & \leqslant 2^{-k} C \int_{0}^{2^{k}} \omega\left(1 /\left(1+r / 2^{j}\right)\right) d r \\
& \leqslant 2^{j-k} C \int_{1 /\left(1+2^{k-j}\right)}^{1} \omega(s) s^{-2} d s .
\end{aligned}
$$

Since $\omega(s)$ is concave, $\omega^{2 / 3}(s)$ is also concave, and hence $\omega^{2 / 3}(s) / s$ is nonincreasing. Thus the last term in the above inequalities is smaller than

$$
\begin{aligned}
C 2^{j-k}\left(1+2^{k-j}\right) \omega^{2 / 3}\left(1 /\left(1+2^{k-j}\right)\right) \int_{0}^{1} \omega^{1 / 3}(s) s^{-1} d s & \\
& \leqslant C \omega^{2 / 3}\left(2^{-|k-j|}\right) \int_{0}^{1} \omega^{1 / 3}(s) s^{-1} d s .
\end{aligned}
$$

Since for $2^{k} \leqslant|z|$ we have $\left(1+2^{k-j}\right)\left(1+2^{-k}|z|\right) \leqslant 3\left(1+2^{-j}|z|\right)$, and since $\omega(t)$ is nondecreasing and $\omega(2 t) \leqslant 2 \omega(t)$ (by the concavity of $\omega$ ), we have

$$
\begin{aligned}
\omega\left(1 /\left(1+2^{-j}|z|\right)\right) & \leqslant C \omega^{2 / 3}\left(1 /\left(1+2^{k-j}\right)\right) \omega^{1 / 3}\left(1 /\left(1+2^{-k}|z|\right)\right) \\
& \leqslant C \omega^{2 / 3}\left(2^{-|k-j|}\right) \omega^{1 / 3}\left(1 /\left(1+2^{-k}|z|\right)\right) .
\end{aligned}
$$


So, noting $j \leqslant k$, we get

$$
\begin{aligned}
\int_{2^{k} \leqslant|z|} p_{j}(z) d z & \leqslant C 2^{-k n} \omega^{2 / 3}\left(2^{-|k-j|}\right) \int_{2^{k} \leqslant|z|} \frac{\omega^{1 / 3}\left(1 /\left(1+2^{-k}|z|\right)\right)}{\left(1+2^{-k}|z|\right)^{n}} d z \\
& \leqslant C \omega^{2 / 3}\left(2^{-|k-j|}\right) \int_{0}^{1 / 2} \omega^{1 / 3}(s) s^{-1} d s .
\end{aligned}
$$

This completes the proof.

\section{References}

[1] G. Bourdaud, $L^{p}$-estimates for certain non-regular pseudo-differential operators, Comm Partial Differential Equations 7 (1982), 1023-1033.

[2] A. P. Calderón, Commutators, singular integrals on Lipschitz curves and applications, in: Proceedings of I. C. M., Helsinki (1978), 85-96.

[3] R. R. Coifman, G. David and Y. Meyer, La solution des conjectures de Calderón, Adv. in Math. 48 (1983), 144-148.

[4] R. R. Coifman and C. Fefferman, Weighted norm inequalities for maximal functions and singular integrals, Studia Math. 51 (1974), 241-250

[5] R. R. Coif man and Y. Meyer, Au delà des opérateurs pseudo-différentiels, Astérisque 57,
Soc. Math. France, 1978. Soc. Math. France, 1978.

[6] G. David, Opérateurs intégraux singuliers sur certaines courbes du plan complexe, Ann. Sci. École Norm Sup. (4) 17 (1984), 157-189.

[7] G. David and J-L. Journé, A boundedness criterion for generalized Calderón-Zygmund operators, Ann. of Math. 120 (1984). 371-397.

[7'] - , -, Une caractérisation des opérateurs intégraux singuliers bornés sur $L^{2}\left(\boldsymbol{R}^{\prime \prime}\right), \mathrm{C} . \mathbf{R}$. Acad. Sci. Paris Sér. I 296 (1983), 761-764. [8] C. Fefferman and E. M. Stein, $H^{p}$ spaces of several variables, Acta Math. 129 (1972),
$137-193$.

[9] R. A. Hunt, B. Muckenhoupt and R. Wheeden, Weighted norm inequalities for the

10] J-L. Journ é, Calderón-Zygmund Operators, Trans. Amer. Math. Soc. 176 (1973), 227-251. J-L. Journé, Calderón-Zygmund Operators, Pseudo-Differential Operators and the Cauchy Integral of Calderón, Lecture Notes in Math. 994, Springer-Verlag, Berlin 1983. [11] Y. Meyer, Sharp estimates for pseudo-differential operators, Proc. Sympos. Pure Math. 35,
Part 2, 1979, 105-113.

[12] N. Miller, Weighted Sobolev spaces and pseudodifferential operators with smooth symbols, Trans. Amer. Math. Soc. 269 (1982), 91-109.

[13] S. Mossaheb and M. Okada, Une classe d'opérateurs pseudo-différentiels bornés sur $L^{r}\left(\boldsymbol{R}^{\prime}\right), 1<r<\infty$, C. R. Acad. Sci. Paris Sér. A 285 (1977), 613-616, and Publications Mathématiques d'Orsay 77-78, 1976-1977, 41-52.

[14] M. Nagase, The $L^{p}$-boundedness of pseudo-differential operators with non-regular symbols, Comm. Partial Differential Equations 2 (1977), 1045-1061.

[15] S. Nis higaki, Weighted norm inequalities for certain pseudo-differential operators, Tokyo
[16] K. Yabuta, Continuity of the mean values of BMO functions and Calderón-Zygmund properties of certain singular integrals, Bull. Fac. Sci. Ibaraki Univ. Math. 15 (1983), 1-8. [17] -, Correction and remark to [16], Bull. Fac. Sci. Ibaraki Univ. Math. 16 (1984), 53-54.

DEPARTMENT OF MATHEMATICS

IBARAKI UNIVERSITY

Received March 19, 1984 